\title{
Adaptation to the climate change: Interest of a prospective approach / Adaptation au changement climatique : l'intérêt d'une démarche prospective
}

\author{
Patrick Aigrain $^{1}$, Françoise Brugière ${ }^{1}$, Eric Duchêne ${ }^{2}$, Inaki Garcia de Cortazar-Atauri ${ }^{3}$, Jacques Gautier ${ }^{4}$, Eric \\ Giraud-Héraud $^{5}$, Hervé Hannin ${ }^{6}$, Nathalie Ollat ${ }^{5}$, et Jean-Marc Touzard ${ }^{7}$ \\ ${ }^{1}$ Etablissement national des produits de l'agriculture et de la mer (FranceAgriMer) Montreuil, France \\ ${ }^{2}$ Institut national de la recherche agronomique (INRA) Colmar, France \\ ${ }^{3}$ INRA Avignon, France \\ ${ }^{4}$ Institut national de l'origine et de la qualité (INAO) Montreuil, France \\ ${ }^{5}$ INRA Bordeaux, France \\ ${ }^{6}$ IHEV Montpellier SupAgro Montpellier, France \\ ${ }^{7}$ INRA Montpellier, France
}

\begin{abstract}
The current challenges of the agronomic research and in particular the adaptation of agriculture to climate change, require a very broad disciplinary mobilization. To meet these challenges, which go beyond its disciplinary and territorial organization, the INRA initiated a new system of interdisciplinary piloting of research. Thus, the métaprogramme ACCAF is trying to understand the joint effects of the various modifications caused by climate change on terrestrial farming and natural environments, and to define adaptation strategies of adaptation as well as their environmental and socio-economic consequences. Within this framework, twenty-three research laboratories have been collaborating in the project LACCAVE and united their efforts in the project LACCAVE in order to examine the effects of climate change on the vine and wine sector. In addition to six disciplinary working groups, this project is made up of an interdisciplinary group including researchers and experts of public institution working with the sector, which has carried out a foresight exercise. By directing the reflection towards a medium-long term future (2030-2050), this prospective exercise authorizes us to leave the temporal horizon of the negotiation and the dictatorship of emergency. As the long-term future is neither known nor recognizable, the evolutions are considered as combinations of assumptions expressed in one potential form and its opposite. For the prospective in the LACCAVE program, four strategies of adaptation of viticulture for 2030-2050 were predefined and a collective and pluridisciplinary work made possible the writing of a plausible way of events leading to each strategy. These results will then be used to debate with the actors of the wine sector at various geographical levels, in order to contribute to the development and the consolidation of choices of strategies of adaptation of the vineyards to climate change. The presentation will focus on this original methodology and its specific implementation. Stories are detailed in another article "Work of prospective on the adaptation of the viticulture to climate change: which series of events could support various adaptation strategies?"
\end{abstract}

\begin{abstract}
A 1'horizon 2050, les vignobles français et leurs filières vont devoir s'adapter au changement climatique, qui modifiera à la fois les conditions de production des vins, leurs caractéristiques organoleptiques et leurs marchés. A cette échéance, d'autres facteurs tels que les évolutions des préoccupations de santé et d'environnement, les changements géopolitiques ou technologiques, les nouvelles conditions de consommation du vin ou encore les modifications de politique agricole affecteront le secteur vitivinicole.

Comment les acteurs de la vigne et du vin pourront-ils s'adapter à un contexte où le changement climatique va se combiner à des changements socio-économiques souvent incertains ? Les leviers d'adaptation envisageables sont multiples : nouveaux cépages, modifications des pratiques viticoles et œnologiques, stratégies de localisation des producteurs et négociants, réorganisation de la filière et
\end{abstract}

de son cadre réglementaire, redéfinition des politiques de recherche et d'innovation... De telles actions sont déjà mises en œuvre ou envisagées, mais souvent sans vision très claire, sans réflexion collective sur des futurs possibles affectés par le changement climatique. Réfléchir et imaginer ces futurs possibles devient stratégique pour les acteurs de la vigne et du vin et pour la Recherche censée les accompagner.

Pour étudier les impacts du changement climatique et les adaptations potentielles du secteur viti-vinicole français, l'INRA a soutenu entre 2012 et 2016 le projet LACCAVE, au sein d'un programme plus large sur l'Adaptation au Changement Climatique de l'Agriculture et de la Forêt (métaprogramme ACCAF). Ce projet a associé des chercheurs de plusieurs disciplines (climatologie, génétique, agronomie, œnologie, économie, sociologie, ....), issus de vingt-trois équipes de l'INRA, 
du CNRS, d'universités et d'écoles d'ingénieurs. (Equipes de recherche des centres INRA de Bordeaux, Montpellier, Avignon, Colmar, Angers, Paris, du CNRS Rennes, de Bordeaux Sciences Agro, de Montpellier SupAgro, des Universités de Limoges, Dijon, Bordeaux.

Une des opérations phare du projet LACCAVE a été la conduite d'un exercice de prospective. Ses objectifs étaient :

- Identifier et explorer différentes stratégies d'adaptation pour la viticulture française dans un contexte de changement climatique, à l'horizon 2050 ;

- Tester une méthode pour élaborer des «chemins » favorisant la mise en œuvre de ces stratégies d'adaptation ;

- Développer une vision commune et des pratiques de travail en réseau entre chercheurs et organisations de la filière.

Cette prospective a été réalisée par une « cellule d'animation » réunissant des chercheurs et des experts d'organismes publics de l'accompagnement de la filière viti-vinicole française (INAO et FranceAgriMer). La cellule a travaillé à l'identification des stratégies d'adaptation envisageables pour le 《système vignes et vins » français face au changement climatique, et à l'élaboration de chemins concourant à la mise en œuvre de ces stratégies.

\section{Les impacts observés et attendus du changement climatique}

Les scénarios d'évolution du climat proposés par le GIEC (Groupement Intergouvernemental d'Experts sur le Changement Climatique) prévoient d'ici la fin du XXI ${ }^{\text {ème }}$ siècle une augmentation de la température moyenne pouvant aller de 2 à $5{ }^{\circ} \mathrm{C}$ selon les hypothèses retenues pour les futures émissions de gaz à effet de serre, avec des variations en fonction des saisons et des régions. (Cf. Fig. 1) Cette augmentation de température va s'accompagner d'un changement du régime des pluies et des besoins en eau des cultures. La variabilité du climat et les événements extrêmes (vagues de chaleurs, pluies intenses...) devraient aussi s'accroître.

L'augmentation de la température enregistrée ces dernières décennies a déjà provoqué des changements observables sur la physiologie de la vigne : avancement de sa phénologie (floraison, véraison) et de la période de récolte (près de deux semaines en moyenne par rapport à la période avant 1980) ; augmentation du degré alcoolique ; baisse de l'acidité du raisin à la récolte ; modification des profils aromatiques et polyphénoliques ; évolution du rendement dans certaines régions... En plus de ces tendances, les viticulteurs ont dû faire face à des événements extrêmes : canicules de 2003 et 2006, printemps très chaud et très sec de 2011, gel en février 2012, été très pluvieux en 2013, hiver 2015-2016 très doux.

L'utilisation de modèles (Travaux réalisées dans le cadre des projets ANR CLIMATOR et ACCAF LACCAVE) permet de simuler les impacts du climat futur sur la vigne et le vin:

- l'avancée de la phénologie devrait se poursuivre, voire s'accélérer dans certaines régions ;

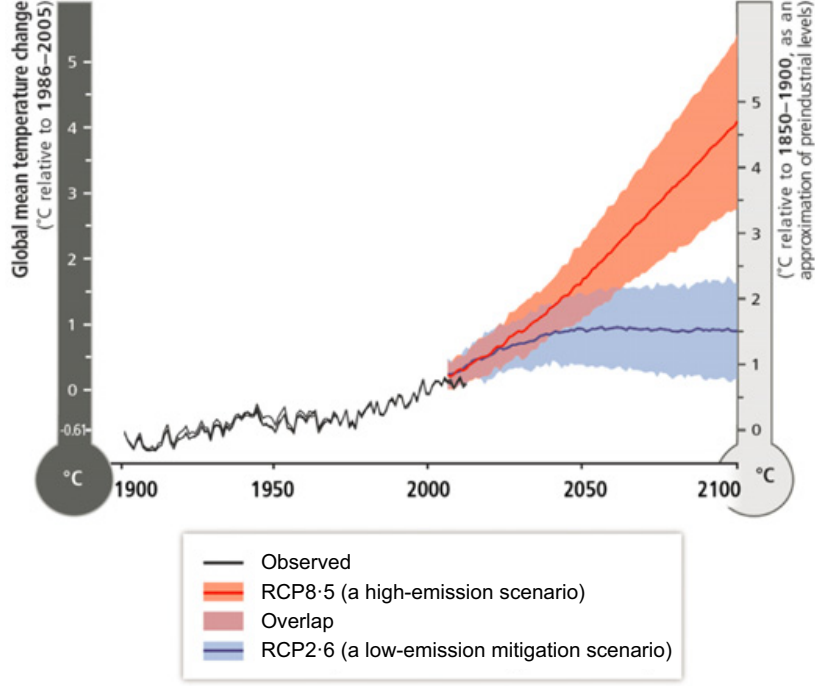

Rapport GIEC 2013

Figure 1. Global average surface temperature change.

- la typicité des vins pourrait changer dans plusieurs régions du fait des nouvelles conditions climatiques durant la période de maturité ;

- la modification du régime des pluies accentuerait les périodes de forte contrainte hydrique dans certaines régions, en particulier pour la partie sud de la France ;

- la production (rendement et composition des raisins) pourrait être affectée de manière très contrastée selon les régions ;

- une augmentation de la pression de maladies cryptogamiques est à envisager, notamment dans les vignobles septentrionaux.

\section{Explorer les solutions pour l'adaptation}

Depuis plus de 2000 ans les viticulteurs ne cessent d'adapter leurs pratiques culturales aux conditions climatiques, mais la vitesse des évolutions actuelles nécessite de nouvelles stratégies. Comme plante pérenne, la vigne apparaît vulnérable à des changements trop rapides. Les conditions et les risques futurs de production du vin doivent donc être largement anticipés.

Dans ce contexte, le projet LACCAVE a exploré plusieurs voies d'adaptation à diverses échelles de temps et d'espace :

- sélection variétale (création de génotypes adaptés aux nouvelles conditions) et changement de variété ou de porte-greffe,

- pratiques culturales à la parcelle (modification de la taille et du rapport feuille/fruit, introduction de l'irrigation, meilleure gestion du sol) ou à la cave (gestion des températures, méthode de désalcoolisation du vin, acidification, levures moins productives en alcool),

- voire relocalisation des parcelles au sein d'une petite région.

Des modifications d'organisation de la filière et de réglementation ont également été considérées, tout comme les investissements à réaliser en recherche, appui technique ou information. Enfin les perceptions des changements 


\section{Proposition de représentation générique et simplifiée du système Vignes-Vins}

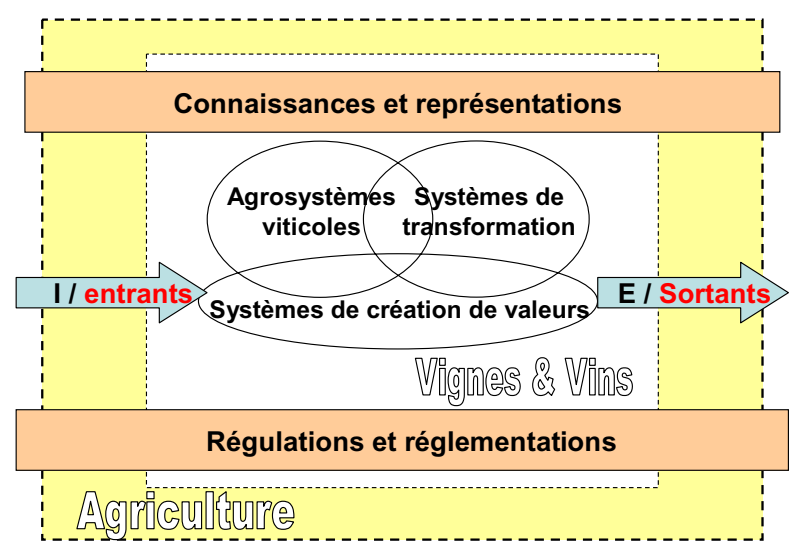

Figure 2. Le système vignes et vins - représentation simplifiée.

par les acteurs du secteur ont été prises en compte, qu'il s'agisse des consommateurs (le vin du « changement climatique » sera-t-il apprécié ?) ou des producteurs (quelles adaptations peuvent être mises en œuvre, et à quelle échéance?).

\section{Une méthode originale de prospective}

Pour conduire cet exercice de prospective, la cellule d'animation a procédé en cinq étapes consistant successivement à :

1 proposer une représentation systémique du secteur vignes et vins ;

2 retenir un scénario climatique et fixer certaines évolutions du contexte ;

3 définir les principales stratégies d'adaptation;

4 recueillir, sélectionner et combiner des hypothèses qui peuvent être liées à ces stratégies ;

5 décrire les chemins possibles permettant la réalisation des stratégies d'adaptation jusqu'en 2050.

\section{1) Représenter le système vignes et vins}

$\mathrm{Au}$ sein de l'agriculture, les activités et flux qui participent à la production et aux échanges du vin peuvent se représenter comme un système, lui-même organisé en trois sous-systèmes : les agrosystèmes viticoles conduisant à la production de raisins, les systèmes de transformation débouchant sur l'élaboration des vins, les systèmes de création de valeur incluant les acteurs économiques de la chaine de valeur, en particulier en aval (négoce, consommateurs, ...). Ces trois sous-systèmes sont dépendants des connaissances et représentations construites à propos de ces activités et de leurs produits. Ils sont également cadrés par les régulations et réglementations du secteur. Par ailleurs des acteurs ou des territoires peuvent entrer ou sortir de ce système. Cf. Fig. 2.

Ce système vignes et vins est doublement concerné par le changement climatique, (cf. Fig. 3) d'une part parce que ce changement agit directement ou indirectement sur ses activités, d'autre part parce que celles-ci contribuent à l'émission de gaz à effet de serre. L'objectif de

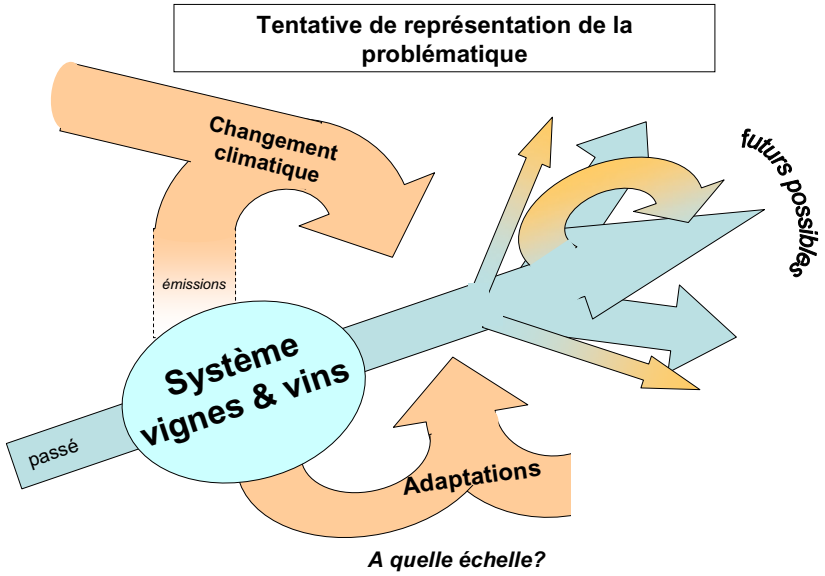

Figure 3. Tentative de représentation de la problématique.

ce travail est l'exploration de voies d'adaptation aux changements climatiques et ne prend pas en compte les possibilités d'atténuation de la contribution du secteur vignes et vins à ces changements climatiques, considérant ici implicitement que ces changements sont largement déterminés par des facteurs extra sectoriels. Ce système va évoluer dans le temps en suivant différents chemins possibles, selon la nature et l'intensité du changement climatique d'une part et d'évolutions socio-économiques - sur lesquelles les acteurs du système vignes et-vins ont quelque prise : par exemple la pression anti-alcool de l'OMS, l'extension des échanges mondiaux à de nouveaux pays, les révisions de la Politique Agricole Commune (PAC) ou encore l'acceptabilité des OGM par les consommateurs... pourraient modifier substantiellement le cadre actuel de production et commerce du vin.

\section{2) Retenir un scénario climatique et des éléments du contexte socio-économique}

Pour réduire la complexité du système et de ses évolutions, la cellule d'animation a retenu un seul scénario climatique pour 2050 et fixé certaines tendances du contexte socioéconomique :

Le choix s'est porté sur un scénario climatique « médian » du GIEC en termes de températures à l'horizon 2050 : entre $+1,5$ et $+2{ }^{\circ} \mathrm{C}$ d'augmentation de la température moyenne (par rapport à l'ère préindustrielle). Cela correspond à une prolongation des tendances récentes, un peu amorties, associées à une augmentation globale de la fréquence d'événements climatiques extrêmes.

Les effets de ce scénario climatique sont variables selon les régions, avec grossièrement un gradient nord-sud :

- dans le nord, si maturité et productivité sont le plus souvent favorisées, le problème réside soit dans l'arrivée de nouveaux bio-agresseurs, soit dans l'accroissement de virulence de bio-agresseurs connus, ce qui complexifie l'obtention d'une production assez stable quantitativement et de qualité aromatique satisfaisante ;

- dans le sud, sécheresse et manque de fraicheur constituent les principaux handicaps, en impactant la régularité du rendement et la viabilité économique. De plus, les vins présentant un degré élevé (naturellement 


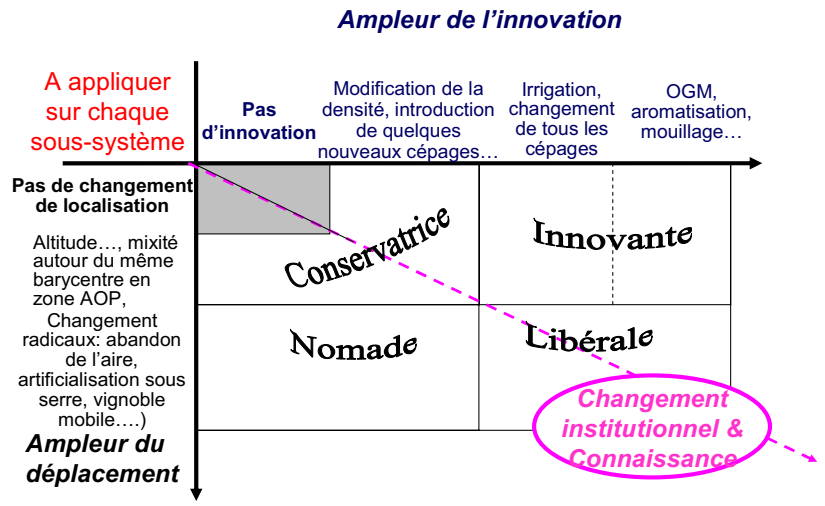

Figure 4. Les stratégies d'adaptation.

obtenu) se révèlent de moins en moins compatible avec les attentes de nombreux consommateurs et des autorités de santé publique.

Par ailleurs des évolutions du contexte socio-économique ont été fixées pour la période 2017-2050 : les vignobles français restent insérés dans un cadre politique national et européen ; des événements exceptionnels (catastrophe, crise majeure, boom économique fulgurant...) sont exclus ; les échanges internationaux et la globalisation restent un mouvement de fond, plus ou moins affirmé ; le vin reste une boisson bien identifiée, mais les pratiques viticoles et œnologiques, l'organisation de la filière, ses réglementations et politiques, les stratégies de ses acteurs ou les attentes de ses consommateurs peuvent fortement varier...

Rappelons que les mesures qui pourraient être prises pour atténuer la contribution à l'effet de serre du secteur vignes et vins au changement climatique n'ont pas été considérées ici.

\section{3) Définir les stratégies d'adaptation}

L'originalité de la méthode a été ensuite de définir des stratégies d'adaptation, en croisant deux axes d'actions, jugés structurants pour le système vignes-vins: (cf. Fig. 4).

a) le choix de localisation des vignes qui peut varier d'un maintien strict dans le périmètre des vignobles actuels, jusqu'à un déplacement important (abandon et création de régions viticoles), en passant par des relocalisations au sein ou aux frontières d'une aire de production.

Cette mobilité permet de retrouver des conditions climatiques « plus favorables » ailleurs (selon l'altitude, la longitude, en tenant compte des types de sols, ...);

b) I'innovation technologique (viticole et œnologique) pouvant avoir différentes intensités, depuis la prolongation d'innovations actuelles, jusqu'à des innovations de rupture (biotechnologies, OGM, association avec d'autres cultures voire avec des panneaux solaires, ... ). Les innovations techniques permettent de modifier le fonctionnement du système vignes et vins en atténuant les impacts ou tirant profit du changement climatique.

Les changements institutionnels et la construction de nouvelles connaissances, qui sont d'autres volets de l'adaptation, ont été considérés comme pouvant être associés à ces deux types d'action.
Le croisement de ces deux axes d'action permet de proposer quatre stratégies d'adaptation :

- la stratégie 《conservatrice» qui n'intègre que des changements à la marge dans les vignobles actuels ;

- la stratégie « innover pour rester» qui ouvre les vignobles à une large gamme d'innovations techniques, permettant de maintenir globalement les localisations actuelles ;

- la stratégie « vignobles nomades» qui donne la priorité à la relocalisation des vignobles en fonction des nouvelles conditions climatiques ;

- la stratégie 《libérale » qui permet de tester une situation où « tout est possible partout ».

Une stratégie d' « adaptation 0 », qui ne ferait évoluer ni les pratiques, ni la localisation, a été écartée. Un système figé, sans réaction aux modifications climatiques, a été jugé trop caricatural, et invalidé par l'histoire viticole faite d'adaptations permanentes mises en œuvre à des rythmes variables.

\section{4) Identifier et combiner les hypothèses}

La cellule d'animation a collecté, sélectionné et organisé des hypothèses qui permettraient le développement de ces quatre stratégies d'adaptation. Les hypothèses correspondent à des processus ou des actions qui peuvent ou non se réaliser, et se décrivent par un énoncé (recto) et son contraire (verso).

Elles ont été constituées à partir de trois sources d'information :

- Les expertises des chercheurs du projet LACCAVE, couvrant de nombreux domaines et disciplines scientifiques

- Exemple d'hypothèse : De nouvelles pratiques œnologiques sont développées et permettent de conserver les caractéristiques actuelles des vins. Versus Il n'y pas d'innovations onologiques qui permettent de conserver les caractéristiques actuelles des vins.

- Les résultats d'une prospective antérieure de l'INRA sur la vigne et le vin (cf bibliographie).

- Exemple d'hypothèse: En Europe, l'espace agricole est l'objet d'une planification poussée où les terres les plus fertiles sont réservées aux cultures vivrières, la vigne étant cantonnée dans des zones de moindre fertilité agronomique. Versus En Europe, l'espace agricole n'est pas l'objet d'une planification poussée, la vigne pouvant être plantée sur les terres les plus fertiles.

- Une série d'entretiens (en 2014 et 2015) avec des responsables du secteur, des négociants et viticulteurs dans trois régions (Bordeaux, Languedoc et Champagne).

- Exemple d'hypothèse: Malgré la pression du changement climatique, l'intégration de l'innovation et l'évolution des pratiques en Appellation d'Origine Protégée (AOP) restent lentes et collectives. Versus Le changement climatique conduit à une accélération de l'intégration des innovations en $A O P$ et à la possibilité d'expérimentations individuelles. 
Soixante-dix hypothèses ont finalement été retenues, puis mises en relation les unes avec les autres dans une matrice d'influence-dépendance selon la méthode SYSPAHMM formalisée par M. Sébillotte (cf bibliographie). Le « modèle » central de la méthode SYSPAHMM est ainsi constitué par une matrice, qui vient consigner les influences de toutes les hypothèses retenues sur les autres, et par là-même alors également les dépendances les unes des autres. Ces influences peuvent être positives (la réalisation d'une hypothèse « $\mathrm{H} 1 »$ favorise celle d'une autre $« \mathrm{H} 2 »$ ) ou négatives (la réalisation d'une hypothèse « $\mathrm{H} 1 »$ défavorise celle d'une autre « $\mathrm{H} 2 »)$ voire nulle (le plus souvent). Le degré d'influence / dépendance de ces hypothèses peut être analysé par un logiciel adapté, HClusterizer, qui en déduit pour l'ensemble des hypothèses une représentation sous la forme d'agrégats dans lesquels les hypothèses incluses sont davantage liées entre elles qu'avec celles des autres agrégats Ces agrégats esquissent alors les squelettes des futurs histoires. Une hypothèse de départ appelée hypothèse motrice, généralement la plus influente sur les autres hypothèses de l'agrégat, est choisie. Ensuite, on s'oblige à écrire une première histoire en la faisant débuter par le recto de cette hypothèse mais aussi une seconde qui commence par le verso de cette même hypothèse. Si on ajoute à cela que certains agrégats appellent deux voire trois hypothèses motrices, on conçoit qu'un agrégat puisse générer quatre ou huit histoires. Les histoires relevant d'agrégats distincts, ne comportent pas d'hypothèses communes.

Le traitement de cette matrice a permis d'agréger les hypothèses et d'écrire 16 «micro-histoires », correspondant à des « briques » pour construire les évolutions du système vigne et vin. La construction d'histoires met en évidence, plus que toutes prévisions, une intelligibilité du système et de ses principales clefs de voûte actuelles. Les micro-histoires sont aussi un moyen privilégié d'établir des ponts entre disciplines et de réduire les effets pernicieux de leur hyperspécialisation.

\section{5) Ecrire une histoire pour chaque chemin}

Ces micro-histoires ont été ensuite reliées de manière cohérente (approche morphologique guidée par l'expertise de la cellule d'animation) pour proposer finalement quatre chemins, conduisant chacun à une stratégie d'adaptation.

Les chemins ont été décrits en organisant un récit à partir des 16 micro-histoires et d'un canevas commun de quatre familles de questions :

- le contexte général du système vignes et vins avec en particulier la politique de santé publique vis-à-vis de l'alcool, les objectifs du financement de la recherche par l'Etat, l'existence ou non d'une gestion publique de l'espace agricole ;

- le contexte viti-vinicole international et plus particulièrement l'évolution de la définition internationale $\mathrm{du}$ vin, les règles internationales d'étiquetage de provenance des vins, la nature et l'évolution de l'intervention publique européenne sur le vignoble (y compris les plantations);

- le contexte viti-vinicole local \& national avec les relations entre la filière et la recherche, les échelles de compréhension des impacts du changement climatique sur la vigne, la capacité structurelle d'adaptation des exploitations, l'attitude des consommateurs face à l'évolution du goût des vins, ou l'évolution de la demande en vins biologiques (en lien avec la dimension internationale);

- les conséquences sur la filière, incluant sa gouvernance (à l'échelle régionale notamment), le poids relatif des régions viticoles, l'évolution de ses performances économique notamment à l'export, le poids des AOP dans les échanges vinicoles.

Chacun des chemins correspond à une combinatoire unique de réponses apportées à ces familles communes de questions.

\section{Conclusion}

Ces stratégies d'adaptation et ces chemins ne sont pas des images prédictives de l'avenir. Ce sont avant tout des outils pour stimuler la réflexion et la concertation des acteurs du secteur vitivinicole, en permettant notamment la prise en compte des interférences entre changement climatique et les autres évolutions possibles du contexte vitivinicole.

\section{Références}

Quénol H. et al., 2014. Changement climatique et terroirs viticoles. Ed. Lavoisier, coll. Tech. \& Doc. 444 p.

Ollat, N., Touzard, J.-M. 2014. Long-term adaptation to climate change in viticulture and enology: Journal International des Sciences de la Vigne et du Vin, numéro spécial LACCAVE

Escudier J.L., Garcia de Cortazar I., Giraud-Héraud E., Le Roux, Ollat N., Quenol H., Touzard J.-M. (2016). Le vignoble français à l'épreuve du changement climatique, La Recherche, 513-514: 60-67, juillet-août 2016

Fuentes Espinoza, A., Giraud-Héraud, E., Pérès, S., Pons, A., Tempere, S. Darriet, Ph, (2016)., Réchauffement climatique et acceptabilité des vins par les consommateurs, Revue des Oenologues, 158, janvier 2016

Touzard J.-M., Ollat N. (2014). Stress hydrique et adaptation au changement climatique pour la viticulture et l'oenologie: le projet LACCAVE. Innovations Agronomiques, 38 (2014) : 131-140

Lebon E, Garcia De Cortazar Atauri I, 2014. Dans un contexte de changement climatique, quels sont les impacts de la sécheresse sur la vigne et sur le devenir des vignobles ? L'exemple du Languedoc. Innovations Agronomiques 38, 1-12

Ollat N, van Leeuwen C, Destrac Irvine A, Marguerit E, Duchêne E, Lebon E, Boursiquot JM, Torregrosa L, 2015. Changement climatique; quels seront les déterminants du choix du matériel végétal? Revue des Enologues 157S, 37-40

Sebillotte, M., Aigrain, P., Hannin, H., Sebillotte, C. 2003. Prospective : Vignes et Vins. Scénarios et défis pour la recherche et les acteurs. Bilan et Prospectives, Inra Editions

Aigrain, P., Hannin, H., Vert J. 2013 Perspectives d'évolution de la filière vignes et vins dans la région Languedoc-Roussillon à l'horizon 2025 CEP Analyses $\mathrm{n} \circ 55$, Agreste

Aigrain, P., Brugière, F., Hannin, H., Touvron, Ch., 2014 Prospective ANIVIN de France. Les Etudes FranceAgriMer 